\title{
Expansão urbana e mobilidade: estudo de caso em Patos - PB
}

\author{
Urban expansion and mobility: a case study in Patos - PB \\ Expansión urbana y movilidad: un estúdio de caso em Patos - PB
}

Recebido: 07/12/2021 | Revisado: 11/12/2021 | Aceito: 20/01/2022 | Publicado: 22/01/2022

\author{
Ana Leticia Ramos Bezerra \\ ORCID: https://orcid.org/0000-0003-1064-2720 \\ Universidade Federal de Campina Grande, Brasil \\ E-mail: annaleticiaramos0@gmail.com \\ Adla Jamilly Vieira Felipe \\ ORCID: https://orcid.org/0000-0002-3384-8935 \\ Universidade Federal de Campina Grande, Brasil \\ E-mail: adlajamilly18@outlook.com \\ Thayse Bezerra da Silva \\ ORCID: https://orcid.org/0000-0002-4933-3389 \\ Universidade Federal de Campina Grande, Brasil \\ E-mail: thaysebes@gmail.com \\ Leonardo Rodrigues Guedes \\ ORCID: https://orcid.org/0000-0002-6739-4919 \\ Universidade Federal de Campina Grande, Brasil \\ E-mail: leoguedesr12@hotmail.com \\ Suelen Silva Figueiredo Andrade \\ ORCID: https://orcid.org/0000-0001-7705-6852 \\ Universidade Federal de Campina Grande, Brasil \\ E-mail: suelen.silva@professor.ufcg.edu.br \\ Larissa Santana Batista \\ ORCID: https://orcid.org/ 0000-0001-5066-521X \\ Universidade Federal de Campina Grande, Brasil \\ E-mail: larissa.batista@ufcg.edu.br
}

\begin{abstract}
Resumo
Nas últimas décadas, as cidades brasileiras tem crescido de forma desenfreada e desordenada, processo este que associado à carência de mobilidade urbana é responsável pela geração de problemas que causam impactos negativos na qualidade de vida da população. Desse modo, levando em consideração a importância da correlação que há entre a expansão e a mobilidade urbana, o planejamento urbano integrado é uma alternativa que pode ser empregada na solução de problemáticas urbanas existentes, além de prevenir que outras surjam. Diante disso, o objetivo desse estudo consiste em investigar a relação que há entre estes processos em uma cidade situada no sertão paraibano, por meio da avaliação da sua mobilidade urbana, utilizando o Î́ndice de Mobilidade Urbana Sustentável (IMUS) e do levantamento de informações sobre como ocorreu o seu crescimento urbano. Assim, o crescimento urbano de Patos favoreceu deslocamentos que se dão por meio de veículos individuais, tendo em vista que não houveram incentivos com o propósito de que a população utilizasse de modos de transporte não motorizados e coletivos. Os resultados demonstram que o índice obtido pode colaborar no desenvolvimento de diagnósticos relevantes. O IMUS calculado para a cidade objeto desse estudo pode ser usado como instrumento capaz de nortear a criação de políticas públicas pautadas na promoção da mobilidade urbana, à medida em que a cidade cresce, a fim de harmonizar essas duas questões do desenvolvimento urbano.
\end{abstract}

Palavras-chave: Mobilidade urbana; Planejamento urbano integrado; Índice de Mobilidade Urbana Sustentável.

\begin{abstract}
In recent decades, Brazilian cities have grown in an uncontrolled and disorderly way, a process that associated with the lack of urban mobility is responsible for the generation of problems that cause negative impacts on the quality of life of the population. Thus, taking into account the importance of the correlation between urban sprawl and mobility, integrated urban planning is an alternative that can be used to solve existing urban problems and prevent others from arising. Therefore, the objective of this study is to investigate the relationship between these processes in a city located in the sertão of Paraiba, through the evaluation of its urban mobility, using the Sustainable Urban Mobility Index (I_SUM) and the survey of information about how its urban growth occurred. Thus, the urban growth of Patos favored displacements that take place by means of individual vehicles, considering that there were no incentives for the population to use non-motorized and collective modes of transportation. The results show that the index obtained can collaborate in the development of relevant diagnoses. The I_SUM calculated for the city that is the object of this study
\end{abstract}


can be used as an instrument capable of guiding the creation of public policies based on the promotion of urban mobility, as the city grows, in order to harmonize these two issues of urban development.

Keywords: Urban mobility; Integrated urban planning; Index of Sustainable Urban Mobility.

\section{Resumen}

En las últimas décadas, las ciudades brasileñas han crecido de forma descontrolada y desordenada, proceso que asociado a la falta de movilidad urbana es responsable de la generación de problemas que causan impactos negativos en la calidad de vida de la población. Así, teniendo en cuenta la importancia de la correlación entre la expansión urbana y la movilidad, la planificación urbana integrada es una alternativa que puede utilizarse para resolver los problemas urbanos existentes y evitar que surjan otros. Por lo tanto, el objetivo de este estudio es investigar la relación entre estos procesos en una ciudad ubicada en el Sertão de Paraiba, a través de la evaluación de su movilidad urbana, utilizando el Índice de Movilidad Urbana Sostenible (IMUS) y el levantamiento de información sobre cómo se produjo su crecimiento urbano. Así, el crecimiento urbano de Patos favoreció los desplazamientos que se producen mediante vehículos individuales, en vista de que no había incentivos para que la población utilizara modos de transporte no motorizados y colectivos. Los resultados muestran que el índice obtenido puede colaborar en la elaboración de diagnósticos relevantes. El IMUS calculado para la ciudad objeto de este estudio puede ser utilizado como un instrumento capaz de orientar la creación de políticas públicas basadas en la promoción de la movilidad urbana, a medida que la ciudad crece, con el fin de armonizar estas dos cuestiones del desarrollo urbano.

Palabras clave: Movilidad urbana; Planificación urbana integrada; Índice de Movilidad Urbana Sostenible.

\section{Introdução}

A medida em que as cidades crescem, tendem a se expandir em torno de suas periferias, devido a ser mais difícil aumentar as densidades centrais (Batty et al., 2003). Enquanto a dimensão espacial do modelo de urbanização disperso está relacionada à uma baixa densidade populacional, um tecido urbano com parcelas caracterizadas por baixa conectividade entre si e, custos elevados na utilização de energia e transportes (Aurambout et al., 2018). O modelo de urbanização compacta, caracterizado por altas densidades urbanas e uso dos solos mistos, possibilita economia de energia, tendo em vista que a eficiência das redes básicas é elevada, bem como do transporte público, utilizando assim, o espaço urbano de maneira mais estratégia e eficiente (Leite \& Awad, 2012).

No cenário atual, pode-se salientar que para além das cidades de grande porte, o prolongamento descontínuo do tecido urbano passou a ser cada vez mais perceptível em centros urbanos de diferentes portes. Nesse sentido, cidades onde antes predominava certa continuidade em seu território, atualmente, tem sua mancha urbana ampliando-se de forma progressivamente descontínua (Reolon \& Miyazaki, 2019). Siqueira (2018) destaca que embora não haja uma concordância em relação ao modelo ideal para uma cidade, o discurso contemporâneo propõe a escolha de soluções que causem menos danos ao meio físico e que a segregação social não seja favorecida na busca por circunstâncias socioambientais melhores para a população urbana.

Nessa perspectiva, segundo Carvalho (2016), desde a metade do século XX, os centros urbanos das nossas cidades vivenciaram um forte e acelerado crescimento populacional. Esse expressivo crescimento, ocorreu, majoritariamente, de maneira desordenada e desregulada, sem que houvesse um desenvolvimento proporcional da infraestrutura de transporte e trânsito. Em consequência dessa deficiência, surgiram vários problemas econômicos, sociais e ambientais que comprometem a qualidade de vida da população.

Dessa forma, a prioridade dada a implantação de infraestrutura para o transporte rodoviário, o prevalecimento do transporte individual em detrimento do coletivo, a falta de incremento dos modos não motorizados e a evidente desarticulação entre o planejamento urbano e de transportes são responsáveis pela realidade desafiadora no que se refere à mobilidade urbana, em que a maioria das cidades se encontra (Costa, 2008).

Assim, a relação de interdependência entre a expansão urbana e mobilidade urbana, mostra-se fundamental para o crescimento das cidades, pois como defende Batty et al. (2003), os sistemas de transportes são essenciais para harmonizar os diferentes padrões de assentamento de uma cidade, desenvolvendo os acessos desde o núcleo até a borda e reduzindo o tempo de deslocamento em áreas compactas (Ewing et al., 2016). 
Nesse contexto, Costa (2008) desenvolveu o Índice de Mobilidade Urbana Sustentável (IMUS), tendo como objetivo diagnosticar as condições de mobilidade e disponibilizar subsídios para o planejamento urbano voltado à promoção da mobilidade urbana sustentável. Por meio dessa ferramenta as cidades de Uberlândia-MG, Itajaí-SC, Curitiba-PR e São CarlosSP efetuaram uma avaliação e diagnóstico das suas condições de mobilidade, como pode ser verificado em Assunção (2012), Dávila (2015), Miranda (2010) e Costa (2008).

Dessa forma, esse trabalho investigou o processo de expansão urbana e a mobilidade urbana em Patos, uma cidade de médio porte e de importância estratégica para a região do Alto Sertão da Paraíba, a fim de compreender a correlação desses fenômenos e constatar a necessidade de integração entre essas duas questões. Ademais, a cidade teve sua mobilidade urbana analisada através da aplicação do IMUS, que consiste em um instrumento de avaliação e controle desenvolvido para orientar o planejamento e gestão da mobilidade ao identificar a situação da mesma no município.

\section{Metodologia}

De acordo com Gerhardt e Silveira (2009), o estudo desenvolvido é caracterizado por ser de natureza aplicada, sendo sua abordagem principal de cunho qualitativo, no entanto, também quantitativo, apresentando como procedimento um estudo de caso para cálculo do Índice de Mobilidade Urbana Sustentável (IMUS), sendo exploratória no que se refere ao objetivo. Quanto a fundamentação teórica, esta explorou as correlações existentes entre expansão urbana e mobilidade urbana descritas na literatura, além de caracterizar a cidade de Patos a partir desses conceitos.

A aplicação do Índice de Mobilidade Urbana Sustentável para Patos seguiu a metodologia desenvolvida por Costa (2008). O IMUS é formado por uma hierarquia de critérios que engloba 9 domínios, 37 temas e 87 indicadores. O sistema de pesos utilizado possibilita a identificação da importância referente a cada critério de forma global e para os enfoques da sustentabilidade no âmbito social, econômico e ambiental. A partir disso, torna-se possível analisar o efeito de ações em diferentes áreas para as três dimensões e constatar os indicadores de maior impacto para os resultados do IMUS, na dimensão global e setorial (Silva et al., 2016).

A avaliação dos indicadores é numérica, variando de 0 a 1 , sendo 0 a pior e 1 a melhor. $O$ grupo de Temas de cada Domínio possui uma pontuação global igual a 1,0 e o grupo de cada Tema também possui uma pontuação global igual a 1,0. A composição do IMUS possibilita também o diagnóstico baseado em um número reduzido de indicadores, nas situações em que o cálculo da totalidade que formam o índice não é possível, devido a inexistência ou indisponibilidade de dados para obtenção desses.

Nesta situação, é necessário redistribuir os pesos dos Indicadores dentro de cada Tema, de modo que o somatório dos mesmos mantenha-se igual a 1,00. Também é possível que esse mesmo processo seja realizado para os Temas dentro dos Domínios, nas situações em que nenhum Indicador do respectivo Tema possa ser calculado (Costa, 2008).

Para a aplicação do IMUS na cidade escolhida é inicialmente realizada a verificação da disponibilidade e qualidade das informações para o cálculo dos indicadores. Segundo Costa (2008), a coleta de dados é realizada por meio de fontes diretas, como a Prefeitura Municipal da cidade, suas secretarias, instituições responsáveis pelo trânsito e transportes, e indiretas a exemplo do site oficial da Prefeitura Municipal, dados e pesquisas realizadas por instituições de ensino e pesquisa, entre outros.

A verificação de disponibilidade da informação diz respeito a demora na obtenção das informações, dessa forma, são classificadas do seguinte modo: curto prazo (CP), médio prazo (MP) e longo prazo (LP). Quanto a qualidade dos dados, a avaliação é feita de acordo com a confiabilidade da informação obtida e são classificadas em alta (A), média (M) e baixa (B), considerando-se também, os seguintes aspectos: atualização, fonte e metodologia utilizada para obtenção dos dados.

O Guia de Elaboração dos Indicadores do IMUS foi utilizado como ferramenta base na obtenção desses, sendo uma parte integrante do IMUS, Costa (2008) elaborou esse documento para que auxiliasse os pesquisadores no cálculo do Índice. O Guia dispõe de informações detalhadas sobre a determinação dos Indicadores que constituem o índice, contendo também o 
Research, Society and Development, v. 11, n. 2, e18211224448, 2022

(CC BY 4.0) | ISSN 2525-3409 | DOI: http://dx.doi.org/10.33448/rsd-v11i2.24448

método de cálculo com instruções para o cálculo desses.

Para a obtenção do resultado final do índice, os indicadores são agregados por meio da média ponderada dos seus scores e pesos. Para o valor final, não existe uma classificação oficializada, entretanto os trabalhos que aplicaram a metodologia do IMUS consideram, de maneira geral, que resultados superiores a média $(0,500)$ são favoravéis, levando em conta que o índice varia de 0 a 1 .

Na Tabela 1 é mostrado como o IMUS é estruturado, com todos os seus respectivos domínios, temas, indicadores e pesos de cada um desses para análise global e setorial (econômica, social e ambiental).

Tabela 1 - Hierarquia de critérios do IMUS e seus respectivos pesos.

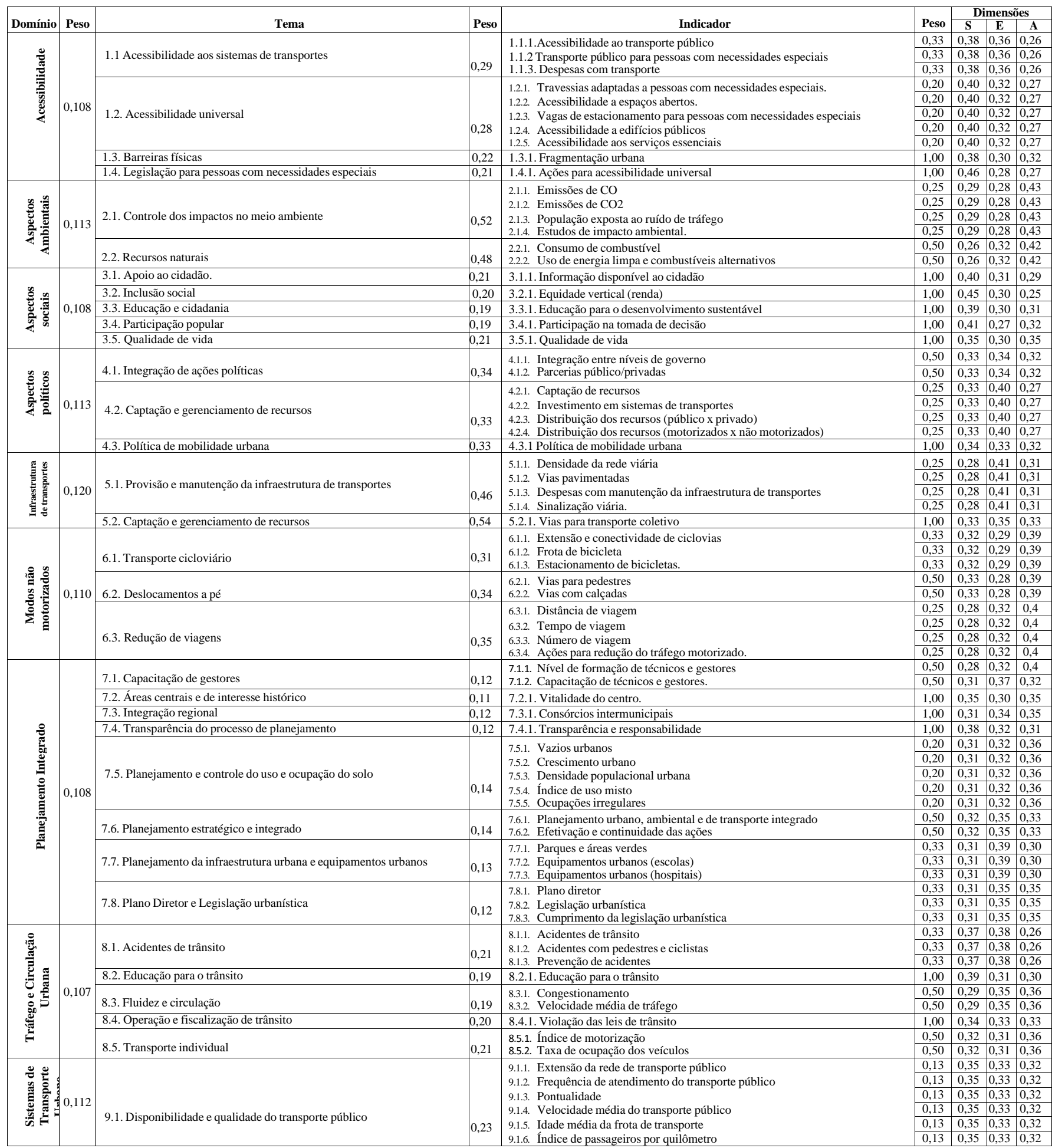




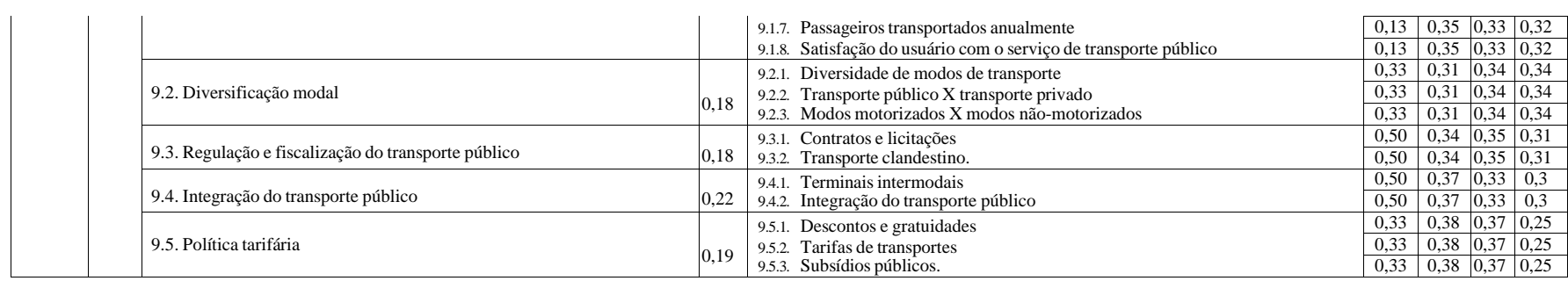

Fonte: Costa (2008).

\section{Resultados e Discussão}

\subsection{Caracterização da Área em Estudo}

Patos é um município brasileiro localizado no estado da Paraíba. Sendo o quarto município mais populoso do estado, possui ainda, a maior população dos munícipios constituintes da mesorregião do Sertão Paraibano, com uma população estimada de 108.192 habitantes, segundo IBGE (2020). Em 2017, o Salário médio mensal dos trabalhadores formais era de 2 salários mínimos, com 15,6\% da população ocupada.

Ademais, Patos é classificada como centro sub-regional A, um nível de categorização da hierarquia dos centros urbanos brasileiros realizada pelo IBGE (2020), sendo definido como nível 3 dos 5 níveis existentes. Conceito esse que engloba cidades responsáveis por exercer influência predominante sobre as cidades próximas, assim, pode-se considerar Patos como uma cidade de grande importância para sua região.

Segundo Lucena (2014), em decorrência da sua posição estratégica, central e a existência de acessibilidade viária, o município de Patos, assumiu historicamente um papel de destaque no contexto do desenvolvimento regional da área em que está inserido, promovendo a cidade à posição de centro intermediário entre cidades maiores e menores, em diferentes redes urbanas estaduais. Além de que, sua localização privilegiada foi responsável por uma centralidade para onde afluem outros municípios da Paraíba e dos estados vizinhos, tornando Patos um centro de comercialização e prestação de serviços com destaque para educação e saúde (Cavalcante, 2008).

Com relação ao sistema de transporte, a cidade conta apenas com os serviços de mototáxi, táxi e táxi lotação, não havendo oferta de transporte público por ônibus desde 2018, quando a empresa que operava o serviço de transporte público encerrou suas atividades. Além disso, o município não dispõe de nenhum terminal urbano de transporte público, o que há na cidade é um terminal de transporte interestadual.

\subsection{Expansão Urbana}

Nas últimas décadas, o município de Patos tem apresentado um expressivo crescimento no que se refere a sua população. Na Tabela 2, é observado o crescimento populacional urbano entre 1991 e 2010, emque consta um crescimento da população urbana, ao passo que a população rural diminuía.

Tabela 2 - População de Patos, 1991 a 2010.

\begin{tabular}{ccccccc}
\hline População & $\mathbf{1 9 9 1}$ & $\mathbf{\%}$ & $\mathbf{2 0 0 0}$ & $\boldsymbol{\%}$ & $\mathbf{2 0 1 0}$ & $\boldsymbol{\%}$ \\
\hline Urbana & 76.708 & 90,6 & 87.949 & 94,3 & 97.278 & 95,8 \\
Rural & 4.590 & 9,4 & 3.812 & 5,7 & 3.396 & 4,2 \\
\hline Total & 81.298 & 100 & 91.761 & 100 & 100.674 & 100 \\
\hline
\end{tabular}

Fonte: IBGE (2010).

A medida em que a população aumentou, a mancha urbana passou por um processo de expansão, pois o crescimento populacional pressupõe a necessidade de construção de novas moradias, equipamentos e serviços para atender as demandas 
da população. Além do mais, no modo de vida da população também ocorreram transformações, isso pode ser observado por um novo perfil pela qual se dão os deslocamentos. O índice de motorização da população (número de veículos para cada 1000 habitantes) cresceu 72,03\% entre 2010 e 2020. Sendo que as principais contribuições para esse aumento, provém da elevação da frota de automóveis e motocicletas, conforme é mostrado na Tabela 3.

Tabela 3 - Evolução da frota de veículos entre 2010 - 2020.

\begin{tabular}{cccccccc}
\hline Tipo/Ano & $\begin{array}{c}\text { Motocicleta/ } \\
\text { Motoneta }\end{array}$ & $\begin{array}{c}\text { Automóvel/ } \\
\text { Camioneta }\end{array}$ & $\begin{array}{c}\text { Ônibus/Micro } \\
\text { Onnibus }\end{array}$ & $\begin{array}{c}\text { Caminhão, Caminhão } \\
\text { trator e } \\
\text { Caminhonete. }\end{array}$ & Outros & $\begin{array}{c}\text { Frota } \\
\text { total }\end{array}$ & $\begin{array}{c}\text { Crescimento } \\
\text { Anual da Frota }\end{array}$ \\
\hline 2010 & 16.472 & 9.397 & 164 & 2.528 & 289 & 28.850 \\
2011 & 19.230 & 10.490 & 176 & 2.751 & 365 & 33.012 & 4.162 \\
2012 & 20.826 & 11.470 & 189 & 2.915 & 413 & 35.813 & 2.801 \\
2013 & 22.312 & 12.500 & 202 & 3.106 & 498 & 38.618 & 2.805 \\
2014 & 23.352 & 13.386 & 214 & 3.243 & 571 & 40.766 & 2.148 \\
2015 & 24.581 & 14.076 & 221 & 3.320 & 1.332 & 43.530 & 2.764 \\
2016 & 25.394 & 14.557 & 222 & 3.388 & 2.297 & 45.858 & 2.328 \\
2017 & 26.233 & 14.956 & 219 & 3.362 & 2.584 & 47.354 & 1.496 \\
2018 & 27.124 & 15.662 & 211 & 3.510 & 2.645 & 49.152 & 1.798 \\
2019 & 28.279 & 16.279 & 223 & 3.630 & 2.764 & 51.175 & 2.023 \\
2020 & 29.121 & 17.138 & 230 & 3.828 & 2.916 & 53.233 \\
\hline
\end{tabular}

Fonte: IBGE Cidades (2020) - Elaborado pelos autores.

Segundo dados do IBGE, entre 2010 e 2020, o número de automóveis aumentou 82,24\% e o de motocicletas 78,85\%. Com o aumento da motorização da população, o uso do transporte individual aumentou, enquanto deslocamentos por meio de modos de transportes não motorizados e coletivos não foram incentivados, isso pode ser constatado pela falta de ciclovias na cidade, falta de acessibilidade adequada nas calçadas e pela inexistência de serviços de transporte público coletivo.

No que se refere ao crescimento espacial da cidade, a dinâmica de formação e expansão da área urbana de Patos seguiu os caminhos que a ligavam às cidades vizinhas, tendo seu crescimento direcionado pelas rodovias que passam pela cidade. Uma vez que o trânsito de veículos e pessoas ao decorrer das vias, atrai a instauração de atividades econômicas e empregos nos arredores dessas. Pois, assim como ressalta Villaça (2001), o tecido urbano é estruturado, fundamentalmente, pelas circunstâncias de deslocamento das pessoas. A Figura 1 demonstra isso. 
Figura 1 - Evolução da mancha urbana entre 1991 - 2020

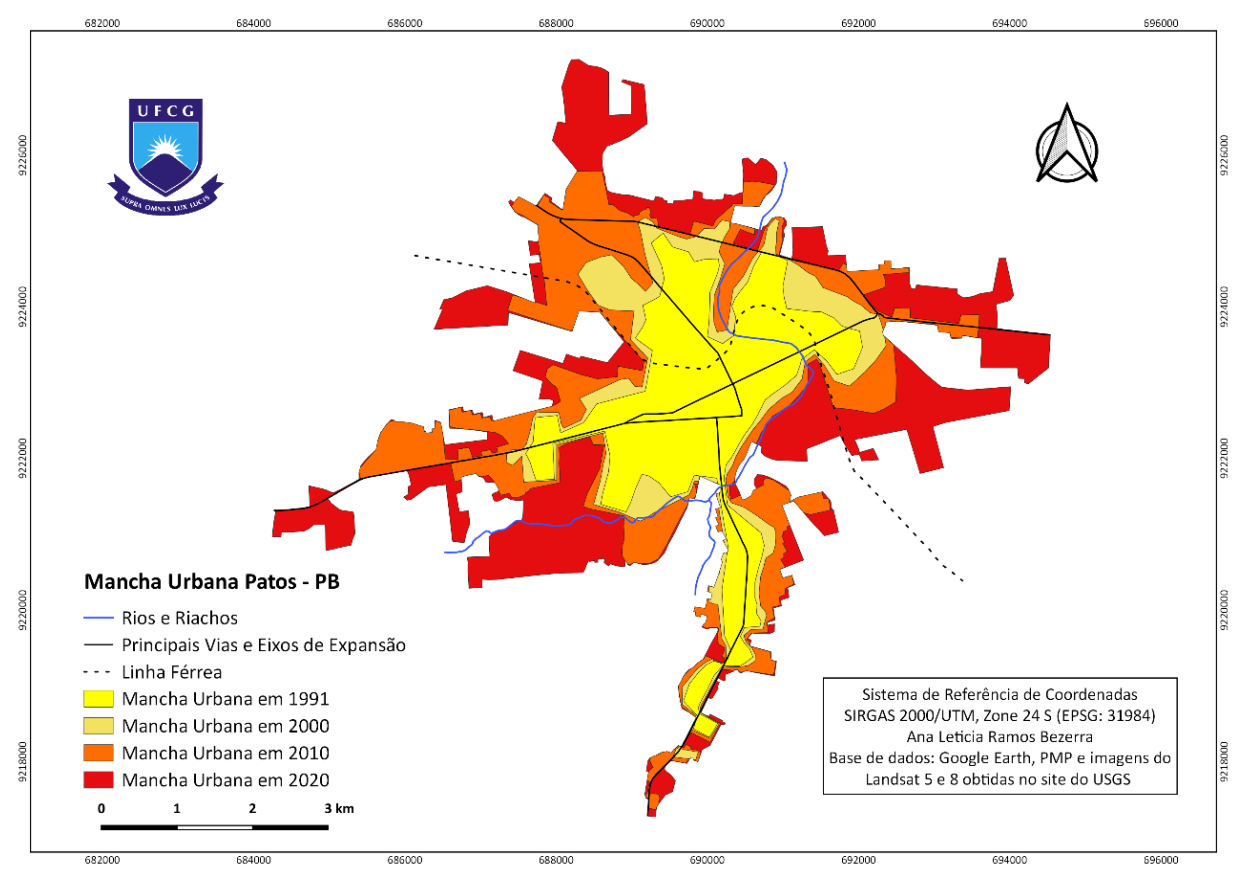

Fonte: Autores (2021).

Nessa perspectiva, analisando o crescimento da mancha urbana do município, foi possível observar que ao decorrer dos anos, houve um espraiamento da cidade, na qual novas áreas foram incorporadas de forma contínua ou não à malha urbana. Dessa forma, seu espraiamento ocorreu no formato disperso, em que a população se espalha para direções mais distantes do centro comercial, desenvolvendo-se de maneira fragmentada, em decorrência da presença de obstáculos a continuidade da malha urbana, como o Rio Espinharas e as rodovias que cortam a cidade, bem como, devido ao fato de que áreas mais distantes receberam algum tipo de construção, enquanto áreas mais próximas não foram desenvolvidas.

\subsection{Avalação da disponibilidade e da qualidade de dados para cálculo do IMUS}

A aplicação do índice de mobilidade urbana sustentável em Patos, foi atencedida pela etapa de avaliação quanto à disponibilidade e qualidade dos dados disponíveis, para o cálculo do índice. Nessa etapa, obteve-se a quantidade de indicadores que poderiam ser obtidos e calculados. As fontes consultadas para obtenção dos dados são apresentadas na Tabela 4.

Tabela 4 - Fontes de dados utilizados na obtenção dos indicadores.

\begin{tabular}{c}
\hline Fontes de dados \\
\hline Agência Nacional de Petróleo (ANP) \\
Câmara Municipal de Patos - Leis Municipais \\
Departamento de Trânsito da Paraíba (DETRAN-PB) \\
Departamento Nacional de Trânsito (DENATRAN) \\
Google Earth e Google Maps \\
Instituto Brasileiro de Geografia e Estatística (IBGE) \\
Levantamentos de campo \\
OpenStreetMap (OSM) \\
Pesquisa de opinião \\
Prefeitura Municipal de Patos \\
Secretaria Municipal de Educação \\
Secretaria Municipal de Infraestrutura e Urbanismo \\
Setor de geoprocessamento da Prefeitura Municipal de Patos \\
Site oficial da Prefeitura Municipal de Patos \\
(STTRANS)
\end{tabular}

Fonte: Bezerra (2021). 
Dos 87 indicadores que formam o IMUS, 56 foram classificados como possíveis de se calcular em curto prazo e com qualidade alta, 1 em curto prazo e qualidade média e 30 indicadores não puderam ser obtidos, pois seu prazo de obtenção foi classificado como longo. Na Tabela 5, é apresentado o percentual da classificação dos indicadores quanto à disponibilidade e qualidade dos dados e de acordo com seu domínio.

Tabela 5 - Percentual da disponibilidade e qualidade dos dados para o cálculo dos indicadores do IMUS referente a Patos.

\begin{tabular}{|c|c|c|c|c|}
\hline \multirow{2}{*}{ Indicadores } & \multicolumn{4}{|c|}{ Classificação } \\
\cline { 2 - 5 } & \multicolumn{2}{|c|}{ Disponibilidade } & \multicolumn{2}{c|}{ Qualidade } \\
\hline Domínios & CP - A & CP - M & CP - B & LP \\
\hline Acessibilidade & $60 \%$ & $0 \%$ & $0 \%$ & $40 \%$ \\
\hline Aspectos Ambientais & $33 \%$ & $17 \%$ & $0 \%$ & $50 \%$ \\
\hline Aspectos Sociais & $80 \%$ & $0 \%$ & $0 \%$ & $20 \%$ \\
\hline Aspectos Políticos & $100 \%$ & $0 \%$ & $0 \%$ & $0 \%$ \\
\hline Infraestrutura de Transportes & $100 \%$ & $0 \%$ & $0 \%$ & $0 \%$ \\
\hline Modos não motorizados & $56 \%$ & $0 \%$ & $0 \%$ & $44 \%$ \\
\hline Planejamento integrado & $95 \%$ & $0 \%$ & $0 \%$ & $5 \%$ \\
\hline Tráfego e circulação urbana & $44 \%$ & $0 \%$ & $0 \%$ & $56 \%$ \\
\hline Sistema de transporte urbano & $33 \%$ & $0 \%$ & $0 \%$ & $67 \%$ \\
\hline Total & $66 \%$ & $1 \%$ & $0 \%$ & $33 \%$ \\
\hline
\end{tabular}

Fonte: Bezerra (2021).

Posteriormente, a verificação da classificação do prazo e qualidade de obtenção dos dados, observou-se que apenas os domínios Aspectos Políticos e Infraestrutura de Transportes tiveram todos os seus indicadores classificados como possíveis de obtenção dos dados a curto prazo e com qualidade alta.

Realizada a etapa de avaliação da disponibilidade e qualidade dos dados para obtenção dos indicadores, chega-se a quantidade de indicadores que podem ser utilizados para o cálculo do índice no município. Para Patos, dos 87 indicadores que compõem o índice, 57 puderam ser calculados, o que equivale a um total de 65,5\% dos indicadores, dividos dentro dos domínios conforme mostra a Figura 2.

Figura 2 - Porcentagem de indicadores calculados para cada domínio.

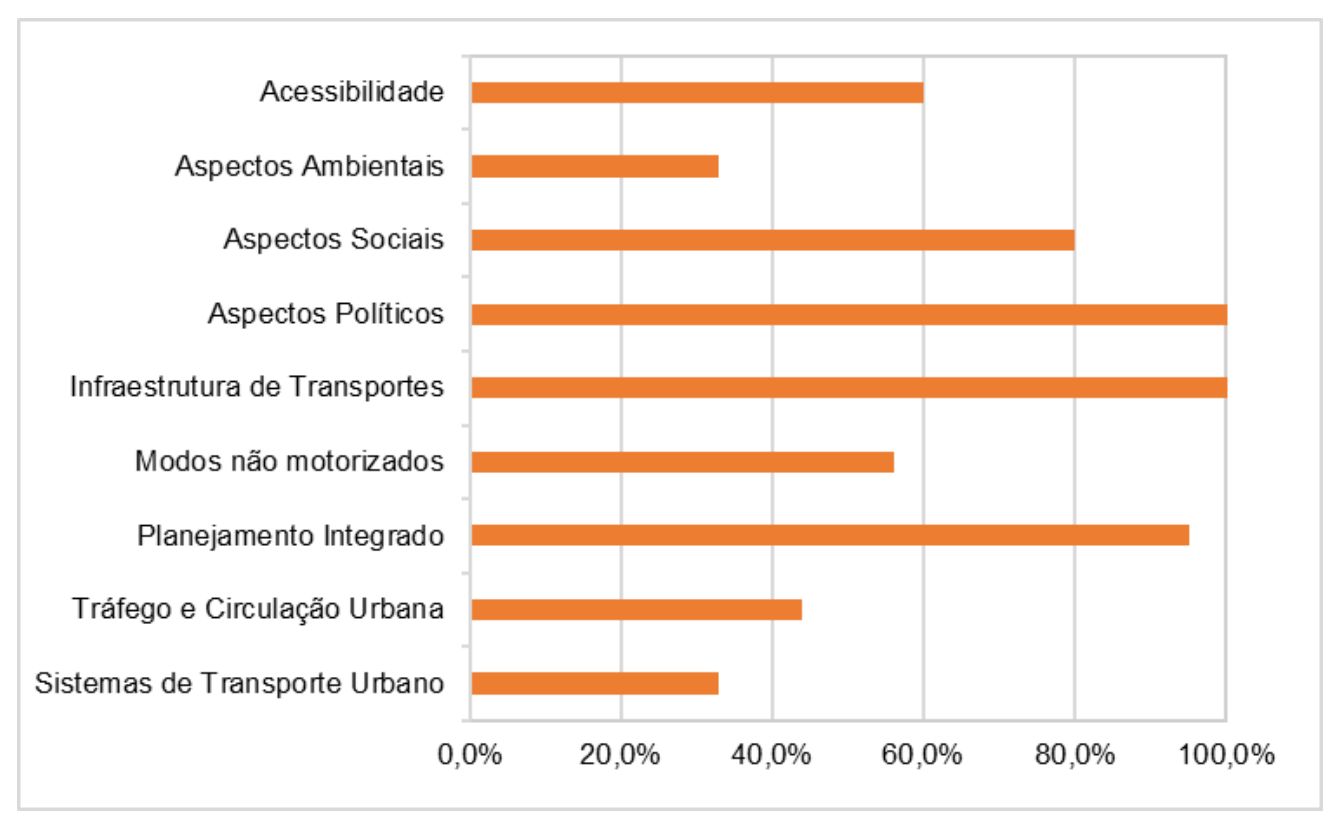

Fonte: Bezerra (2021). 
De tal forma, a desconsideração de vários indicadores realizada para o cálculo do IMUS referente a Patos é reflexo da indisponibidade ou falta de levantamento de dados por parte dos orgãos públicos da cidade, da inexistência do serviço de transporte público por ônibus, bem como da ausência de uma pesquisa Origem e Destino.

\subsection{Cálculo do IMUS e desempenho dos indicadores}

O Índice de Mobilidade Urbana Sustentável para a cidade de Patos foi calculado a partir da obtenção de 57 indicadores dos 87 que compõem o índice. O resultado do IMUS global é 0,389, valor abaixo da média (0,500).

Tabela 6 - Resultado do IMUS Patos.

\begin{tabular}{lcc}
\hline \multirow{2}{*}{ IMUS } & \multicolumn{2}{c}{ RESULTADO } \\
\cline { 2 - 3 } & Absoluto & Corrigido \\
\hline IMUS $_{\text {global }}$ & 0,280 & 0,389 \\
IMUSs $_{\text {ocial }}$ & 0,090 & 0,125 \\
IMUS $_{\text {Econômico }}$ & 0,095 & 0,128 \\
IMUS $_{\text {Ambiental }}$ & 0,095 & 0,136 \\
\hline
\end{tabular}

Fonte: Bezerra (2021).

Quanto ao valor do IMUS para Patos, são apresentados os resultados absolutos e corrigidos, em que foi realizada uma redistribução dos pesos dos indicadores e temas não obtidos. Os valores setoriais e que representam as dimensões da sustentabilidade apresentados na Tabela 6 podem atingir valores máximos de 0,34, 0,33 e 0,33.

Os valores alcançados para cada indicador estão apresentados de forma agrupada por domínio na Tabela 7 . Os indicadores que obtiveram valor máximo $(1,00)$ estão destacados em verde, correspondendo a 13 no total. Para estes indicadores é recomendado empenhar-se em sua manutenção para que seus bons resultados sejam conservados. Os indicadores marcados em vermelho obtiveram valor mínimo $(0,00)$ e, por isso, são considerados críticos, sendo 17 no total. Estes indicadores são aqueles que deverão ser imediatamente analisados pela gestão pública da cidade, para que sejam solucionados. Já os indicadores denominados "vazios", não puderam ser calculados devido à indisponibilidade de informações.

Tabela 7 - Resultado dos indicadores agrupados por domínio.

\begin{tabular}{|c|c|c|c|c|}
\hline Domínio & Indicador & P. & P.R. & $\mathrm{S}$ \\
\hline \multirow{10}{*}{ 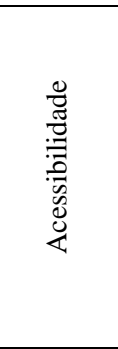 } & Acessibilidade ao transporte público & 0,33 & 0,50 & 0,00 \\
\hline & Transporte público para pessoas com necessidades especiais & 0,33 & 0,50 & 0,00 \\
\hline & Despesas com transportes & 0,33 & - & Vazio \\
\hline & Travessias adaptadas para pessoas com necessidades especiais & 0,20 & 0,50 & 0,00 \\
\hline & Acessibilidade aos espaços abertos & 0,20 & - & Vazio \\
\hline & Vagas de estacionamento para pessoas com necessidades especiais & 0,20 & 0,50 & 0,20 \\
\hline & Acessibilidade a edifícios públicos & 0,20 & - & Vazio \\
\hline & Acessibilidade aos serviços essenciais & 0,20 & - & Vazio \\
\hline & Fragmentação urbana & 1,00 & 1,00 & 0,00 \\
\hline & Ações para acessibilidade universal & 1,00 & 1,00 & 0,25 \\
\hline \multirow{6}{*}{ 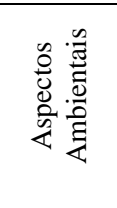 } & Emissões de $\mathrm{CO}$ & 0,25 & - & Vazio \\
\hline & Emissões de $\mathrm{CO} 2$ & 0,25 & - & Vazio \\
\hline & População exposta ao ruído de tráfego & 0,25 & - & Vazio \\
\hline & Estudos de impacto ambiental & 0,25 & 1,00 & 0,75 \\
\hline & Consumo de combustível & 0,50 & 0,5 & 1,00 \\
\hline & Uso de energia limpa e combustíveis alternativos & 0,50 & 0,5 & 0,11 \\
\hline \multirow{5}{*}{ 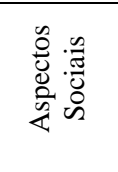 } & Informação disponível ao cidadão & 1,00 & 1,00 & 0,50 \\
\hline & Equidade vertical (renda) & 1,00 & - & Vazio \\
\hline & Educação para o desenvolvimento sustentável & 1,00 & 1,00 & 0,75 \\
\hline & Participação na tomada de decisão & 1,00 & 1,00 & 0,33 \\
\hline & Qualidade de vida & 1,00 & 1,00 & 0,80 \\
\hline \multirow{4}{*}{ 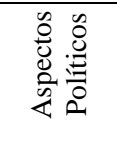 } & Integração entre níveis de governo & 0,50 & 0,50 & 0,75 \\
\hline & Parcerias público-privadas & 0,50 & 0,50 & 1,00 \\
\hline & Captação de recursos & 0,25 & 0,25 & 1,00 \\
\hline & Investimentos em sistemas de transporte & 0,25 & 0,25 & 0,25 \\
\hline
\end{tabular}


Research, Society and Development, v. 11, n. 2, e18211224448, 2022

(CC BY 4.0) | ISSN 2525-3409 | DOI: http://dx.doi.org/10.33448/rsd-v11i2.24448

\begin{tabular}{|c|c|c|c|c|}
\hline & Distribuição dos recursos (coletivo x privado) & 0,25 & 0,25 & 0,00 \\
\hline & Distribuição dos recursos (motorizado x não motorizado) & 0,25 & 0,25 & 0,25 \\
\hline & Política de mobilidade urbana & 1,00 & 1,00 & 0,25 \\
\hline \multirow{5}{*}{ 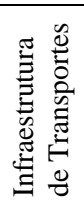 } & Densidade e conectividade da rede viária & 0,25 & 0,25 & 1,00 \\
\hline & Vias pavimentadas & 0,25 & 0,25 & 0,52 \\
\hline & Despesas com manutenção da infraestrutura & 0,25 & 0,25 & 0,50 \\
\hline & Sinalização viária & 0,25 & 0,25 & 0,60 \\
\hline & Vias para transporte coletivo & 1,00 & 1,00 & 0,00 \\
\hline \multirow{9}{*}{ 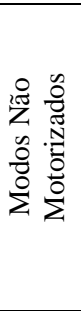 } & Extensão e conectividade de ciclovias & 0,33 & 0,5 & 0,00 \\
\hline & Frota de bicicletas & 0,33 & - & Vazio \\
\hline & Estacionamento para bicicletas & 0,33 & 0,5 & 0,00 \\
\hline & Vias para pedestres & 0,50 & 0,50 & 0,00 \\
\hline & Vias com calçadas & 0,50 & 0,50 & 0,17 \\
\hline & Distância de viagem & 0,25 & - & Vazio \\
\hline & Tempo de viagem & 0,25 & - & Vazio \\
\hline & Número de viagens & 0,25 & - & Vazio \\
\hline & Ações para redução do trafego motorizado & 0,25 & 1,00 & 0,25 \\
\hline \multirow{18}{*}{ 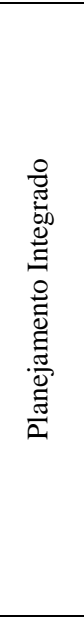 } & Nível de formação de técnicos e gestores & 0,50 & 0,50 & 1,00 \\
\hline & Capacitação de técnicos e gestores & 0,50 & 0,50 & 0,00 \\
\hline & Vitalidade do centro & 1,00 & 1,00 & 0,50 \\
\hline & Consórcios intermunicipais & 1,00 & 1,00 & 0,00 \\
\hline & Transparência e responsabilidade & 1,00 & 1,00 & 0,25 \\
\hline & Vazios urbanos & 0,20 & 0,25 & 0,88 \\
\hline & Crescimento urbano & 0,20 & - & Vazio \\
\hline & Densidade populacional urbana & 0,20 & 0,25 & 0,00 \\
\hline & Índice de uso misto & 0,20 & 0,25 & 1,00 \\
\hline & Ocupações irregulares & 0,20 & 0,25 & 1,00 \\
\hline & Planejamento urbano, ambiental e de transportes integrado & 0,50 & 0,50 & 0,33 \\
\hline & Efetivação e continuidade de ações & 0,50 & 0,50 & 1,00 \\
\hline & Parques e áreas verdes & 0,33 & 0,33 & 0,00 \\
\hline & Equipamentos urbanos (escolas) & 0,33 & 0,33 & 1,00 \\
\hline & Equipamentos urbanos (postos de saúde) & 0,33 & 0,33 & 1,00 \\
\hline & Plano Diretor & 0,33 & 0,33 & 0,50 \\
\hline & Legislação urbanística & 0,33 & 0,33 & 0,50 \\
\hline & Cumprimento da legislação urbanística & 0,33 & 0,33 & 0,75 \\
\hline \multirow{9}{*}{ 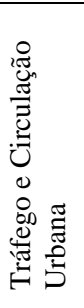 } & Acidentes de trânsito & 0,33 & - & Vazio \\
\hline & Acidentes com pedestres e ciclistas & 0,33 & - & Vazio \\
\hline & Prevenção de acidentes & 0,33 & - & Vazio \\
\hline & Educação para o trânsito & 1,00 & 1,00 & 0,14 \\
\hline & Congestionamento & 0,50 & 0,50 & 1,00 \\
\hline & Velocidade média do tráfego & 0,50 & - & 1,00 \\
\hline & Violação das leis de trânsito & 1,00 & - & Vazio \\
\hline & Índice de motorização & 0,50 & 1,00 & 1,00 \\
\hline & Taxa de ocupação de veículos & 0,50 & & Vazio \\
\hline \multirow{18}{*}{ 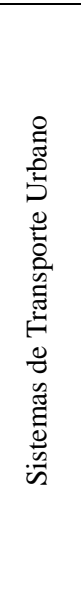 } & Extensão da rede de transporte público & 0,13 & 1,00 & 0,00 \\
\hline & Frequência de atendimento do transporte público & 0,13 & - & Vazio \\
\hline & Pontualidade & 0,13 & - & Vazio \\
\hline & Velocidade média do transporte público & 0,13 & - & Vazio \\
\hline & Idade média da frota de transporte público & 0,13 & - & Vazio \\
\hline & Índice de passageiros por quilômetro & 0,13 & - & Vazio \\
\hline & Passageiros transportados anualmente & 0,13 & - & Vazio \\
\hline & Satisfação do usuário com o serviço de transporte público & 0,13 & - & Vazio \\
\hline & Diversidade de modos de transporte & 0,33 & 1,00 & 0,25 \\
\hline & Transporte coletivo $\mathrm{x}$ transporte individual & 0,33 & - & Vazio \\
\hline & Modos não-motorizados x modos motorizados & 0,33 & - & Vazio \\
\hline & Contratos e licitações & 0,50 & 0,50 & 0,00 \\
\hline & Transporte clandestino & 0,50 & 0,50 & 0,25 \\
\hline & Terminais intermodais & 0,50 & 1,00 & 0,00 \\
\hline & Integração do transporte público & 0,50 & - & Vazio \\
\hline & Descontos e gratuidades & 0,33 & - & Vazio \\
\hline & Tarifas de transporte & 0,33 & _- & Vazio \\
\hline & Subsídios públicos & 0,33 & 1,00 & 0,00 \\
\hline
\end{tabular}

Fonte: Bezerra (2021).

As contribuições dos domínios para o resultado final do IMUS foram respectivamente: Tráfego e circulação urbana (19,40\%), favorecido pela boa fluidez na circulação dos veículos e índice de motorização da população adequado. Aspectos Ambientais (19,04\%), em decorrência do consumo per capita de gasolina apresentar bons resultados, Aspectos Sociais (16,50\%), 
favorecido, principalmente, pela elevada porcentagem de entrevistados,, que classificou Patos como um lugar bom ou excelente para se viver e pela existência de ações no que se refere à educação para o desenvolvimento sustentável. Aspectos políticos $(14,62 \%)$, em razão dos recursos obtidos por meio de multas e taxações, serem revertidos em investimentos para transporte e mobilidade, e por parcerias público-privadas de projetos de mobilidade ja terem sido implementadas no município.

Planejamento integrado (13,70\%), favorecido, principalmente, por uma quantidade expressiva dos técnicos e gestores da Superintendência de Transito e Transportes de Patos, possuírem qualificação em nível superior pela permição de usos mistos do solo em todo área urbana, pelas ocupações irregulares ocuparem uma área pequena da cidade e por Patos possuir um bom número de escolas e postos de saúde.

Colaborando de modo intermediário está o domínio Infraestrutura (9,28\%), prejudicado, principalmente, pela inexistência de vias para transporte coletivo na cidade. O desempenho desse domínio é reflexo da necessidade de maiores investimentos na infraestrutura viária da cidade.

Os domínios que apresentaram resultados piores foram: Modos não motorizados $(3,29 \%)$, Acessibilidade $(2,23 \%)$ e Sistemas de Transporte Urbano (1,94\%), com uma grande quantidade de indicadores que alcançaram valores críticos, sendo reflexo da falta de incetivos aos modos não motorizados, a exemplo da inexistência de ciclovias na cidade, falta de acessibilidade adequada para deslocamentos de pessoas com deficiências e inexistência de transporte público por ônibus.

Sendo assim, os resultados alcançados podem colaborar no desenvolvimento de medidas, por parte da administração municipal, que levem em consideração a distribuição de intervenções para melhoria da mobilidade urbana de acordo com os domínios do IMUS. A Figura 3, apresenta a contribuição de cada domínio para o valor final do IMUS, como também mostra uma comparação entre os resultados obtidos para cada domínio e valores máximos possíveis para esses.

Figura 3 - IMUS Patos e IMUS Máximo

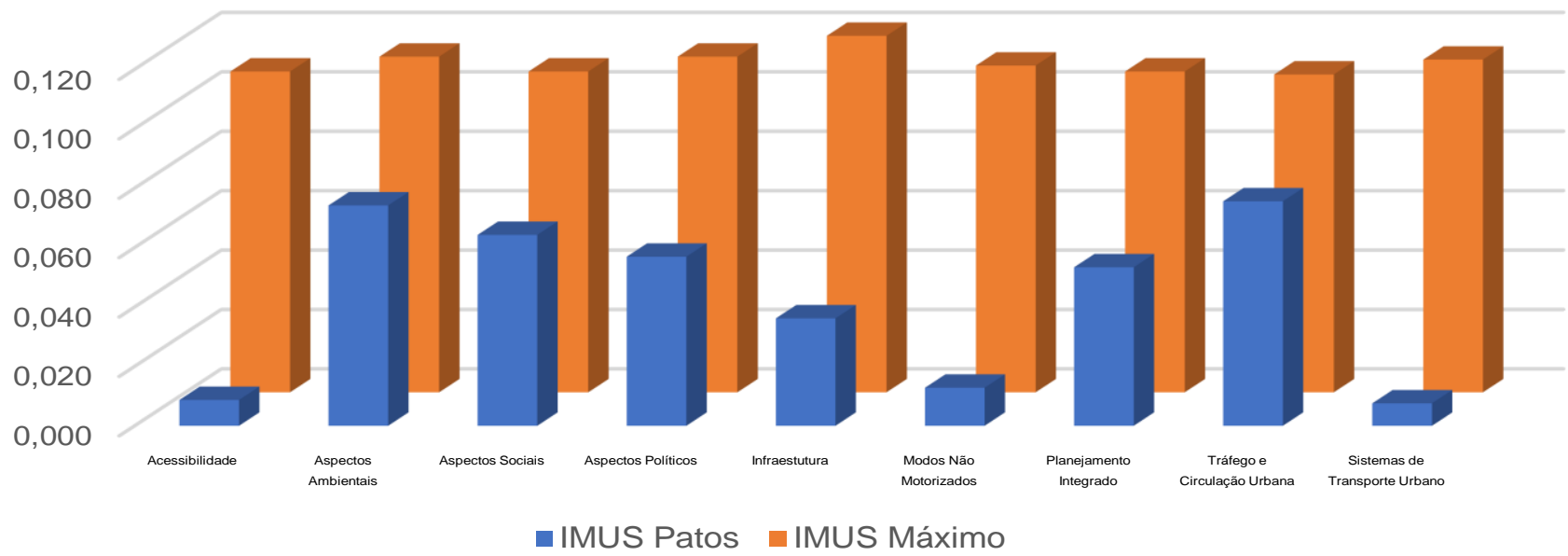

Fonte: Bezerra (2021).

Entre os indicadores críticos, pode-se observar que 7 deles mostram como Patos se desenvolveu por meio de políticas que priorizam a utilização do transporte individual, em detrimento da inexistência de oferta de transporte público coletivo e falta de incetivo ao uso de modos de transportes não motorizados, realidade essa que alerta para necessidade de tornar a mobilidade urbana prioritária no que se refere ao planejamento urbano da cidade. Sendo os indicadores mencionados: acessibilidade ao transporte público, transporte público para pessoas com necessidades especiais, extensão e conectividade de ciclovias, estacionamento para bicicletas, vias para pedestres, extensão da rede de transporte público e subsídios públicos.

Como reflexo da indisponibilidade de transporte público em Patos, 20 indicadores alcançaram valores críticos ou não puderam ser obtidos. Sendo que desses, 7 obtiveram valores críticos (acessibilidade ao transporte público, transporte público 
para pessoas com necessidades especiais, distribuição dos recursos (público x privado), vias para transporte coletivo, extensão da rede de transporte público, contratos e licitações, e subsídios públicos) e 13 não puderam ser calculados (despesas com transporte, crescimento urbano, frequência de atendimento do transporte público, pontualidade, velocidade média do transporte público, idade média da frota de transporte, índice de passageiros por quilomêtro, passageiros transportados anualmente, satisfação do usuário com o serviço de transporte público, transporte público x transporte privado, integração do transporte público, descontos e gratuidades, e tarifas de transporte).

Quanto a forma pelo qual se deu a expansão urbana da cidade, indicadores do IMUS, como vazios urbanos, densidade populacional urbana e fragmentação urbana, podem ser utilizados para caracterizar a dispersão de sua área urbana. Em Patos, os vazios urbanos representam 14,66\%, a densidade populacional é de 3.896 habitantes $/ \mathrm{km}^{2}$, sendo encontradas 23 subdivisões na área efetivamente urbanizada. Entretanto, Costa (2008) considera como valores ideias que os vazios urbanos correspondam em até $10 \%$ da área urbana, densidade de 45.000 hab $/ \mathrm{km}^{2}$ e quanto a fragmentação urbana, a não existência de subdivisões na área urbana da cidade.

Demonstrada a correlação existente entre a mobilidade urbana e expansão urbana, é de extrema importância que essas temáticas sejam incluídas de maneira integrada na concepção das políticas e legislações da cidade. Para assim, fazer com que o desenvolvimento urbano ocorra com espaços urbanos utilizados de forma otimizada, garantindo qualidade ambiental, além de dispor de acessibilidade para a pessoa com deficiência, a fim de que a cidade seja um ambiente urbano harmônico e sustentável, e assim, ofereça qualidade de vida aos cidadãos.

\subsection{IMUS como ferramenta de comparação}

No processo de diagnóstico da mobilidade realizado por meio do IMUS, a comparação dos resultados alcançados com o de outras cidade que já tiveram sua mobilidade avaliada é importante, para assim, situar a cidade em relação a sua mobilidade urbana e analisar fatores que podem ser melhorados para alcançar um resultado final de melhor desempenho. Sendo assim, na Tabela 8 é apresentado uma comparação entre o IMUS calculado para Patos e os de outras cidades que utilizaram esse método.

Tabela 8 - Comparação entre resultados do IMUS.

\begin{tabular}{lccccc}
\hline \multicolumn{1}{c}{$\begin{array}{c}\text { Dimensão do } \\
\text { IMUS }\end{array}$} & Patos & Uberlândia & Itajaí & Curitiba & São Carlos \\
\cline { 2 - 6 } & 0,389 & 0,717 & 0,572 & 0,748 & 0,561 \\
\hline IMUS $_{\text {global }}$ & 0,125 & 0,243 & 0,195 & 0,255 & 0,189 \\
\hline IMUSS $_{\text {ocial }}$ & 0,128 & 0,236 & 0,188 & 0,248 & 0,188 \\
\hline IMUS $_{\text {Econômico }}$ & 0,136 & 0,238 & 0,188 & 0,245 & 0,185 \\
\hline IMUS $_{\text {Ambiental }}$ & &
\end{tabular}

Fonte: Adaptado de Assunção (2012), Dávila (2015), Miranda (2012) e Costa (2008).

A observação da tabela permite verificar que Patos se encontra em uma posição abaixo das cidades analisadas, com destaque para Curitiba que apresentou o melhor resultado $(0,748)$. No entanto, vale ressaltar que os indicadores calculados se diferenciam de cidade para cidade.

O desempenho da análise de cada cidade individualmente, é mostrado na Figura 4, considerando os resultados alcançados para cada um dos nove domínios que compõem o IMUS. 
Figura 4 - Desempenho das cidades de São Carlos, Curitiba, Itajaí, Uberlândia e Patos em cada domínio.

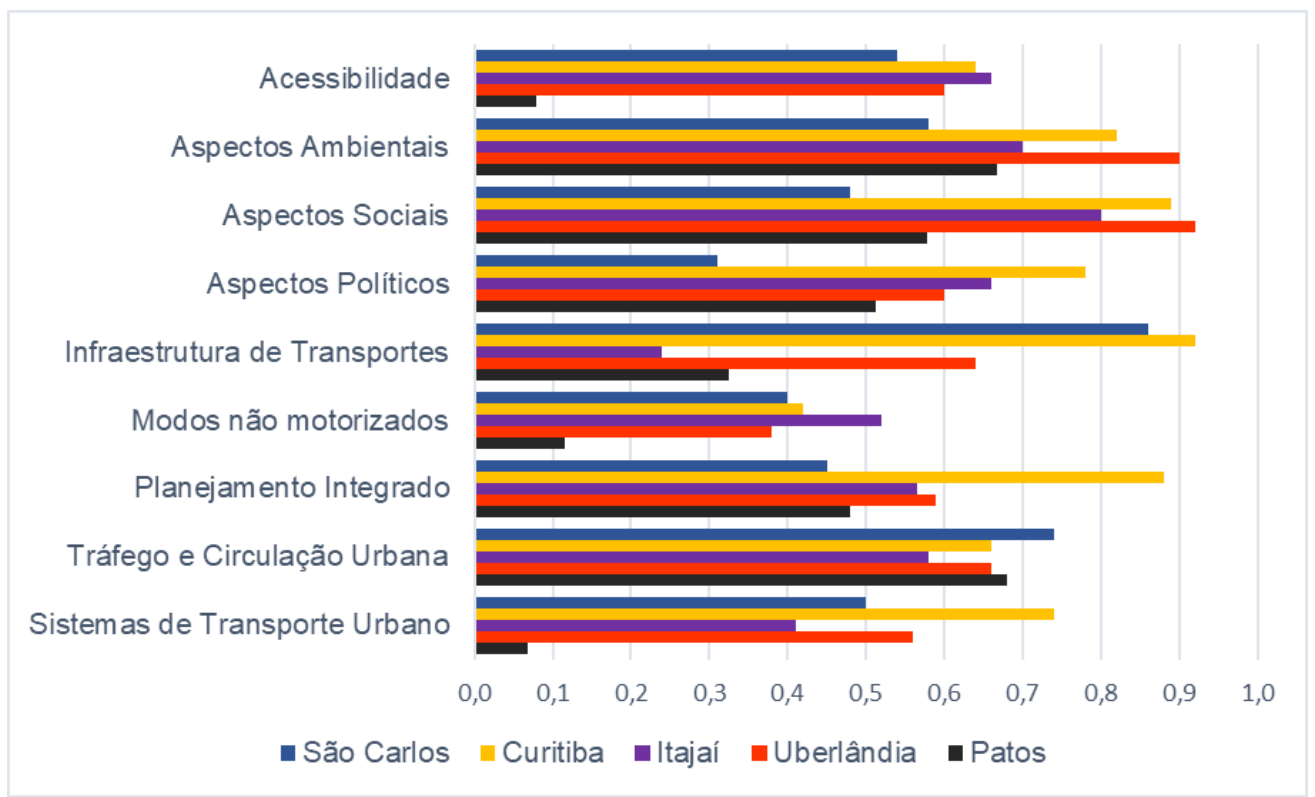

Fonte: Adaptado de Costa (2008), Miranda (2010), Dávila (2015) e Assunção (2012) .

Ainda que as cidades comparadas sejam de maior porte que Patos, o IMUS como ferramenta de comparação serve como exemplo, para que os gestores sigam o modelo de planejamento e gerenciamento da mobilidade urbana de cidades que obtiveram um bom desempenho no cálculo do índice, seja desenvolvendo políticas públicas baseadas em experiências bem sucedidas ou reproduzindo intervenções urbanas eficazes.

\section{Conclusão}

Pode-se concluir que, Patos é classificado como centro sub-regional A, conceito que engloba cidades responsáveis por exercer influência predominante sobre as cidades próximas.

O processo de expansão urbana de Patos seguiu os caminhos que ligam o munícipio às cidades vizinhas, tendo em vista que seu crescimento foi direcionado pelas rodovias que cortam a cidade. Quanto à forma em que se deu o espraiamento da mancha urbana, foi possível observar que ocorreu no formato disperso, pois enquanto a população se espalhou para direções mais distantes do centro comercial, áreas mais próximas não foram ocupadas.

Dos 87 indicadores que compõem o IMUS, foi possível calcular 57 indicadores para o IMUS referente a Patos. O resultado alcançado foi de 0,389 . Como o valor obtido foi inferior à média de 0,5 , em tese, o resultado a qual se chegou foi desfavorável. De tal forma, é necessário que haja mais atenção do poder público quanto a questão da mobilidade urbana no município.

Em outro aspecto, a expansão urbana de Patos privilegiou modos de transporte individuais, de forma que não houve incentivos efetivos e eficazes para que a população utilizasse de modos de transporte não motorizados e coletivos em seus deslocamentos, seja pela falta de desenvolvimento de uma infraestrutura adequada para os modos não motorizados ou falta de subsídios públicos para o transporte público.

No âmbito do planejamento integrado, a atualização do Plano Diretor de Patos e, a elaboração e implantação da Política de mobilidade urbana do município, mostram-se de caráter emergencial, pois esses instrumentos de planejamento urbano representam enorme relevância para o desenvolvimento de uma cidade, já que consistem em elementos que são responsáveis pelo delineamento e direcionamento da mobilidade. 
Além disso, a realização de uma pesquisa Origem e Destino com a finalidade de quantificar e caracterizar o padrão de viagens da cidade é de fundamental importância para que, por meio de seus resultados, seja possível dispor de dados que contribuam em planos e projetos futuros de mobilidade urbana.

A tentativa de levantamento de informações que os órgãos públicos ou instituições da cidade não dispunham, revela a necessidade do poder público em deter dados necessários ao monitoramento e avaliação da mobilidade urbana no município.

Dessa forma, o IMUS pode ser utilizado como um instrumento para nortear a concepção de políticas públicas pautadas no desenvolvimento da mobilidade urbana e de um planejamento urbano integrado entre a expansão urbana e do sistema de transporte, à medida que a cidade cresce. Atentando-se ainda para aspectos que envolvem uma melhor inclusão social, no que se refere a harmonização de diferentes padrões de assentamento na cidade e maiores incentivos a utilização de modos de transporte não motorizados e coletivos, além de investimentos em acessibilidade para a população acometida por deficiências e em uma melhor infraestrutura viária para cidade.

\section{Referências}

Assunção, M. A. (2012). Indicadores de mobilidade urbana sustentável para a cidade de Uberlândia, MG. Dissertação de mestrado, Universidade Federal de Uberlândia - UFCG.

Aurambout, J. P., Barranco, R. \& Lavalle, C. (2018). Towards a Simpler Characterization of Urban Sprawl Across Urban Areas in Europe. Land. 7(33). https://doi.org/10.3390/land7010033

Batty, M., Besussi, E. \& Chin, N. (2003). Traffic, urban growth and suburban sprawl. Centre for Advanced Spatial Analysis, n. 70, University College London. https://discovery.ucl.ac.uk/id/eprint/216

Bezerra, A. L. R (2021). Aplicação do índice de mobilidade urbana sustentável (IMUS) na avaliação da mobilidade de Patos. (Trabalho de conclusão de curso). Universidade Federal de Campina Grande, Pombal, 2021.

Carvalho, C. H. R. de. (2016). Mobilidade Urbana: avanços, desafios e perspectivas. In: Costa, M. A. (Org.). O Estatuto da Cidade e a Habitat III: um balanço de quinze anos da política urbana no Brasil e a Nova Agenda Urbana. (pp. 345-361). Brasília, DF: IPEA.

Cavalcante, V. L. U. (2008). A centralidade da cidade de Patos-PB: Um estudo a partir de arranjos espaciais. (Dissertação de mestrado). Universidade Federal da Paraíba, João Pessoa, 2008.

Costa, M. S. (2008). Um índice de mobilidade urbana sustentável. (Tese de doutorado). Universidade de São Paulo, São Carlos, 2008.

Dávila, G. P. (2015). Avaliação da mobilidade urbana em Itajaí. (Trabalho de Conclusão de Curso). Universidade Federal de Santa Catarina, Joinville, 2015.

Ewing, R., Hamidi, S., Grace, J. B. \& Wei, Y. D. (2016). Does urban sprawl hold down upward mobility? Landscape and Urban Planning. 148, 80-88. https://www.sciencedirect.com/science/article/pii/S016920461500242X?via\%3Dihub.

Gerhardt, T. E., \& Silveira, D. T. (2009). Métodos de pesquisa. Editora da UFRGS.

Instituto Brasileiro de Geografia e Estatística (IBGE). (2000). Censo Demográfico (2000). https://www.ibge.gov.br.

Instituto Brasileiro de Geografia e Estatística (IBGE). (2010). Censo Demográfico (2010). https://www.ibge.gov.br.

Instituto Brasileiro de Geografia e Estatística (IBGE). (2020). IBGE Cidades. https://cidades.ibge.gov.br/brasil/pb/patos/pesquisa/22/28120

Instituto Brasileiro de Geografia e Estatística (IBGE). (2020). Regiões de influência das cidades 2018. https://biblioteca.ibge.gov.br/visualizacao/livros/liv101728.pdf

Instituto Brasileiro de Geografia e Estatística. (2020). Estimativa Populacional. https://cidades.ibge.gov.br/brasil/pb/patos/panorama.

Leite, C., \& Awad, J. C. M. (2012). Cidades sustentáveis, cidades inteligentes: desenvolvimento sustentável em um planeta urbano. BOOKMAN.

Lucena, W. G. (2014). A produção do espaço urbano da cidade de Patos/PB: do BNH ao Programa Minha Casa Minha Vida. (Dissertação de mestrado). Universidade Federal da Paraíba, João Pessoa, 2014.

Miranda, H. F (2010). Mobilidade urbana sustentável e o caso de Curitiba. Dissertação de mestrado, Universidade de São Paulo, São Carlos, 2010.

Reolon, C. A. \& Miyazaki, V. K. (2019). Urbanização, dispersão das cidades e aglomeração urbana: um olhar sobre as cidades médias. Revista Terra Plural, Ponta Grossa. 13, 55-72. http://dx.doi.org/10.5212/TerraPlural.v.13i3.0006

Silva, A. N. R., Costa, M., \& Macêdo, M. H. (2016). Planejamento integrado, organização espacial e mobilidade sustentável no contexto de cidades brasileiras. In: Balbim, R., Krause, C., \& Linke, C. C. (Orgs.). Cidade e Movimento. (pp. 81-100). DF: IPEA.

Siqueira, R. A (2018). O adensamento no espaço urbano: as promessas da cidade compacta. (Tese de doutorado). Universidade Federal de Minas Gerais, Belo Horizonte, 2018.

Villaça, F. (2001). Espaço intra-urbano no Brasil. Studio Nobel: FAPESP: Lincoln Institute, 